\title{
Diferenciación de la astronomía: Autodescripciones y concepción heliocéntrica en el horizonte de la sociedad mundial
}

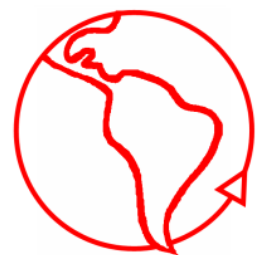

\author{
Differentiation of Astronomy: Self-descriptions and Heliocentric \\ Conception on the Horizon of World Society
}

Alejandro Espinosa Rada

Becario Conicyt del programa de Magíster en Sociología, Pontificia Universidad Católica de Chile

\section{RESUMEN}

La sociogénesis de la astronomía moderna se imbrica espacio-temporalmente con tres innovaciones en la formación de la sociedad mundial entre el siglo XV y XVI: la diferenciación funcional, la membrecía organizacional y las tecnologías de la comunicación. Este hecho puede ser rastreado a través de distintos textos y/o modelos cinemáticos como auto-descripciones de la sociedad, que intentan dar una explicación empírica y/o teórica a los movimientos retrógrados de los planetas. Estos son: el modelo de Eudoxo de Cnido, la síntesis aristotélica, el modelo de Ptolomeo, el modelo de Copérnico y la consolidación del heliocentrismo tras las observaciones de Galileo y la gravitación universal de Newton. Este problema de identidad en la astronomía, junto con producir la diferenciación de la astronomía, cambia la autodescripción de la sociedad en vías de mundialización confiriendo una sociogénesis moderna.

PALABRAS CLAVE: astronomía; heliocentrismo; diferenciación funcional; sociedad mundial; autodescripciones

\section{ABSTRACT}

Sociogenesis of modern astronomy overlaps spatio-temporally with three innovations in the core of world society between the XV and XVI century: functional differentiation, organizational membership and communication technologies. This can be explored throughout different texts and/or kinematic models such as self-descriptions of society that try to give an empirical and/or theoretical overview to the retrograde motions of the planets. These are: the model of Eudoxus of Cnidus, the Aristotelian synthesis, the model of Ptolemy, Copernican heliocentrism model and the consolidation of heliocentrism after the observations of Galileo and Newton's universal gravitation. This identity problem in astronomy along with producing differentiation of astronomy changes the self-description of society in the sociogenesis of world society.

KEYWORDS: astronomy; heliocentrism; functional differentiation; world society; self-descriptions

\section{INTRODUCCIÓN}

Sócrates consideraba que la "astronomía" ( $\alpha \sigma \tau \rho o v o \mu i ́ \alpha)$ tenía como principio el estudio del movimiento de los astros, del sol y la luna, y sus velocidades relativas. El interés principal estaba vinculado a la reforma del

\section{REVISTA MAD - UNIVERSIDAD DE CHILE}

Revista del Magíster en Análisis Sistémico Aplicado a la Sociedad

Facultad de Ciencias Sociales, Departamento de Antropología.

Universidad de Chile

www.revistamad.uchile.cl 
calendario y el tiempo, como lo hicieron los antiguos Babilónicos, por ello, el movimiento de los cuerpos celestes era crucial para la temprana astronomía (Torreti 2007: 34-35). Sin embargo, un largo proceso socioevolutivo derivó en que se modificara la forma de entender el movimiento de estos cuerpos, cambiando a su vez la forma en que la sociedad se entendía a sí misma. Este lugar del hombre en el mundo, se correspondió con el cambio de una noción geocéntricamente situada hacia otra heliocéntrica.

Si consideramos que la sociedad no puede conocerse desde afuera como grupos de seres humanos o como territorios, sino que esta es entendida desde adentro (Luhmann 2007), entonces podemos suponer que todas las observaciones de la sociedad son realizadas en la sociedad. Este último supuesto implica descubrirse a sí mismo en el propio objeto, aprender de él y presume, a su vez, trasladar las ideas a la realidad (y por tanto, asume también que las ideas de otros sean interpenetradas). Es, en síntesis, una auto-implicación cognitiva en las descripciones que se generan, donde se entremezcla semántica y estructura. No obstante, para que las semánticas se estructuren como condensación de expectativas, debe haber un extenso proceso socio-evolutivo anterior.

La ciencia es probablemente uno de los casos más paradigmáticos para rastrear este proceso. En particular, el paso de una noción geocéntrica del mundo a otra heliocéntrica permite desentrañar la forma en que esto se vuelve posible. Para ello, se propone hacer una breve revisión de la historia de la astronomía hasta el siglo XVII. Para Luhmann (2009: 63-64) en particular, la complejidad del mundo desde la perspectiva astronómica, geográfica y cultural sacudió las idas acerca de la perfección del mundo, provenientes de la tradición escolástica orientada por Aristóteles. Por ello, la principal hipótesis que se plantea es que la diferenciación funcional de la astronomía es el resultado de un problema de identidad, el cual debe efectuarse mediante las operaciones del sistema y se exponen a la autorreferencia del sistema, en este caso, el problema astronómico en el sistema de la astronomía o, como señala Luhmann, "Por eso, más que otros sistemas funcionales, la ciencia se diferencia por los problemas que resultan de su propia comunicación" (1996: 442). La diferenciación de la astronomía, a su vez, coincide con el origen de la sociedad mundial al concordar con tres innovaciones que permiten la formación de las estructuras de esta sociedad (Stichweh 2012a, 2012b): la diferenciación funcional, la membrecía organizacional y la imprenta. Con estas tres innovaciones de la socie- 
dad mundial, entremezcladas con los problemas propios de la astronomía (como parte del sistema de la ciencia) se puede rastrear la autodescripción de la posición relativa del hombre en el mundo. En otras palabras, una re-entry de la sociedad en el sistema de la ciencia.

Se entenderá que la auto-observación es una operación dirigida dentro del sistema hacia el sistema, mientras que las autodescripciones son las elaboraciones de textos correspondientes con dichas observaciones (Luhmann 2007). Por ello, para poder realizar este breve recorrido por la historia de la astronomía, se tomarán como puntos de referencia ciertos textos fundamentales: las escrituras cuneiformes de los babilónicos, la " $F^{\prime}$ sica" de Aristóteles, el "Almagesto" de Ptolomeo, el libro "Sobre las revoluciones de las esferas celestes" de Copérnico y los "Principia" de Newton. Estas son algunas obras que (entre otras) situaron la ciencia normal (Kuhn 2008), entendido esto último, como la conformación de paradigmas que surgen por pequeños hitos inaugurales que luego son institucionalizados por comunidades de científicos y discípulos, los que luego entran en conflicto acérrimo con otras teorías contrapuestas, las que finalmente (si salen victoriosas) tienden a estabilizarse. Me limitaré sin embargo a las dos primeras revoluciones científicas para identificar este proceso sociogenético que, según Kuhn (2008), son las de Copérnico y Newton (las otras dos son las de Lavoisier y Einstein, pese a que para un grupo restringido de profesionales, las ecuaciones de Maxwell fueran tan revolucionarias como las ecuaciones de Einstein). Estos libros condensaron sentidos que fueron conservados para facilitar procesos de autorreferencia, en donde adquirieron, en varios casos, sentidos normativos y los cuales compensan un carácter de singularidad y de acontecimiento. Para este caso, se intenta rastrear el proceso socio-evolutivo que da forma a la astronomía diferenciada de la religión (funcionalmente diferenciada en el siglo XVII), sin pretender detallar las contribuciones particulares en los planteamientos astronómicos en cada caso. Tampoco consideraré las contradescripciones de la astronomía provenientes de la astrología (las cuales podrían rastrearse desde Egipto, pasando por ejemplo por el tetrabiblos de Ptolomeo) o los argumentos de la teología (como podría profundizarse en el caso de Santo Tomás de Aquino), pues realizar aquello, supondría considerar un acoplamiento estructural con otros sistemas y/o revisar con mayor detención otros problemas de identidad propios del sistema de la ciencia. Se considera, sin embargo, que esta aproximación empírica contribuirá a hacerse un mapa sobre la relación entre sociedad y astronomía en código sistémico. Por lo tanto, lo que se pretende hacer a continuación es identi- 
ficar algunos sucesos en el proceso socio-evolutivo de la sociedad que permitan dar cuenta de la incipiente sociogénesis de la astronomía diferenciada, lo que derivó en un cambio radical en la autodescripción de la sociedad, pasando de una noción geocéntrica del mundo a otra heliocéntrica.

\section{AUTORREFERENCIA EN LA ASTRONOMÍA}

La sociedad y los individuos, no ha tenido una descripción única sobre la relación entre la tierra y el universo. Los primeros registros de la historia de la astronomía datan de la antigua Babilonia (2000 a.C. - 200 a.C.) producto de tablas con escrituras cuneiformes. En dichas tablas se encuentra el registro de las primeras predicciones astronómicas, al igual que las primeras manifestaciones conocidas sobre las matemáticas (al igual que en Egipto), concebidas para la agricultura y la astronomía (Bell 2012: 36 - 37). No obstante, la verdad del conocimiento astronómico se tematizaba como la pretensión de predecir (por acumulación de datos) fenómenos celestes, tales como: eclipses lunares y solares, los días en que la luna nueva era visible por primera vez, el cálculo de la distancia del sol y sus posiciones en el zodiaco (franja por donde pasa el sol conocida como la eclíptica) (Kahn 1970: 103-104). Las auto-observaciones del espacio eran concebidas desde el punto de vista del observador, quien observaba el lento movimiento relativo de las "estrellas fijas" (cambio relativo por día, semana, mes, de lo que se entendía como "bóveda celeste"), comparándolas a su vez con el movimiento de otras "estrellas errantes" (planetas) que se movían más rápido (conjunciones entre astros). Sin embargo, la principal característica de la astronomía babilónica era que no se hacía la distinción entre fenómenos climáticos y celestes, y se buscaba predecir, pero no necesariamente explicar teóricamente. En esta cosmovisión existía un firmamento geocéntricamente situado.

Por otro lado, en la antigua Grecia se cambió la forma de comprender el universo. Los griegos fueron los primeros que generaron modelos teóricos para realizar representaciones de los astros utilizando objetos cotidianos. Estos modelos correspondían a una observación de segundo orden que situaba desde afuera al observador (reducía complejidad con la utilización de modelos). Con ello se intentó explicar la relación de los astros entre sí, a diferencia de los babilónicos que no intentaba explicar ne- 
cesariamente, sino que predecir. Este cambio paradigmático generó las primeras teorías sobre el universo.

La principal característica de esta forma de conocimiento consistía en que el comportamiento terrestre servía para explicar los fenómenos celestes. Dos escuelas de pensamiento serán relevantes para la astronomía: en primer lugar estaban los jonios ${ }^{1}$, quienes intentaban explicar los principios ( $\left.\dot{\rho} \rho \eta^{\prime}\right)$ que componían a los astros. En segundo lugar, se encontraban los pitagóricos, que a diferencia de los primeros, intentaron proporcionar leyes o axiomas (dinámicas) del universo, junto con considerar que existía una simetría radial que explicaba por primera vez la noción esférica del universo (así como el sol, la tierra, la luna y las estrellas). Además, los pitagóricos consideraban que "lo más digno" (por tener motivos religiosos) era el centro que no era la tierra, sino una bola de fuego más allá de las estrellas fijas, junto con considerar las relaciones geométricas del universo con cualidades armónicas (acústica), donde se vinculan la geometría con la aritmética (los principios estaban en los números y figuras) tal como se aprecia en el tetraktys y las figuras oblongas (Africa 1961; Clarke 1962; Kahn 1970; Goldstein y Bowen 1983). No es de extrañar la influencia de los pitagóricos en la transmisión del conocimiento durante la Edad Media (Luhmann 2007: 753), en donde se distinguía entre trívium (gramática, retórica y dialéctica) y quadrivium (aritmética, geometría, astronomía y música). Sin embargo, la forma en que los pitagóricos comprendían la relación entre sociedad y universo consistía en una visión no-geocéntrica del mundo, pero prontamente, esta apreciación cae en olvido y/o simplemente no fue considerada.

Posteriormente Platón, junto con entregarle una gran relevancia a la astronomía ${ }^{2}$, construye el primer modelo del cosmos que progresivamente se va modificando, además de señalar que el movimiento de las estrellas era por motivos religiosos (la Necesidad y sus hijas Parcas). Sin embargo, los problemas aparejados a explicar fenómenos concretos, como el movi-

\footnotetext{
${ }^{1}$ Un caso interesante es el de Anaxágoras (500 aprox. - 428 aprox. a.C.) quien es uno de los primeros en considerar que los astros eran rocas y quien consideraba que la tierra era plana al igual que Anaxímenes (588 aprox. - 524 aprox. a.C.), junto con ser el primero en plantear la hipótesis del éter ("aire celeste" que mueve los astros). Sin embargo, la consideración de la tierra plana no era compartida por todos los jonios, como es el caso de Anaximandro (618 aprox. - 545 aprox. a.C.). Otro jonio digno de mención es Empédocles (495 - 435 a.C.) quien consideraba que el sol era una mancha concentrada y pulida en una superficie interna, que reflejaba un fuego externo.

${ }^{2}$ De dos de sus discípulos, Euclides (geometría plana) y Teeteto (geometría tridimensional o "sólida"), Platón se quejaba de la baja relevancia que adquiría la geometría sólida en comparación a la geometría euclidiana en Atenas, considerando que la primera era un área fundamental para el estudio astronómico.
} 
miento retrógrado de marte, generó refinamientos posteriores. Ello fue una preocupación de Eudoxo de Cnido (408 - 355 a.C.), discípulo de Platón, que produjo el primer modelo cinemático (Torreti 2007: 21) que explicaba el movimiento retrógrado de $\mathrm{Marte}^{3}$. Como puede apreciarse, es la "anomalía de marte" (extraños movimientos de un planeta, que no tiene un desplazamiento tan armónico como los demás) la que genera problemas en las descripciones astronómicas.

Por otro lado, será Aristóteles quien intenta generar una teoría del universo y la naturaleza, realizando una primera gran síntesis de las distintas ramas del conocimiento (terrestre, biológico, astronómico, teológi$\mathrm{co}$, entre otros). En su síntesis teórica que prevalece hasta alrededor de 1600 (Torreti 2007: 49), el modelo anteriormente realizado por Eudoxo se amplía a 55 esferas (más satisfactorio desde un punto teológico), además la causa del movimiento celeste se la atribuye a un motor inmóvil y en donde las "causas últimas" estaban afuera y se transmitían hacia adentro. Esta apreciación es fundamental, pues dicho motor (y luego dirá que son vario motores según los movimientos circulares independientes) se correspondía con "el dios" como ser vivo, eterno y óptimo, y con una vida eterna (Torreti 2007: 52-53). Por ello, en el modelo aristotélico, se dividió el mundo entre un mundo sublunar (mutable, con cuatro materias fundamentales y con un tipo de movimiento natural que era limitado y vertical) y un mundo superlunar (inmutable, con la quintaesencia del éter ${ }^{4}$ y con un tipo de movimiento natural que era sin fin y circular), junto con considerar a la tierra esférica (y no elíptica). Esta primera gran teoría de la sociedad sitúa semánticamente dos mundos claramente identificables junto con aportar elementos teológicos en las autodescripciones del mundo, en ella varias áreas del saber se unifican en una gran síntesis del cosmos.

Durante cierto periodo, la astronomía gozaba de una gran relevancia en las esferas del conocimiento, sin embargo por largos años esta primacía se vio desplazada por la astrología (300 a.C. - 200 d.C.). En varios siglos se realizaron diversas contribuciones al conocimiento, que no cam-

\footnotetext{
${ }^{3}$ Platón propone un modelo de 8 esferas, mientras que Eudoxo lo amplía a 27 esferas utilizando en sus explicaciones una curva llamada hipopede. Es interesante señalar que las esferas eran entendidas como superficies geométricas sin espesor y con el mismo radio, o sea, configuraciones cinemáticas. Sin embargo, los conceptos matemáticos fueron considerados como abstracciones de la materia.

${ }^{4}$ Para Aristóteles, era necesario considerar el éter, dado que consideraba que la fuerza era proporcional a la masa por la velocidad (donde Newton difiere). A su vez, la velocidad era considerada como inversamente proporcional a la resistencia y que, por tanto, si el vacío tenía una resistencia $=0$ entonces la velocidad era infinita y esto era considerado como absurdo, por tanto debía existir algún tipo de resistencia y para ello se utilizaba el éter.
} 
biarían significativamente las preposiciones aristotélicas, salvo casos excepcionales que generaron grandes contribuciones para la astronomía ${ }^{5}$, pero que en términos de auto-descripción de la sociedad no modificaron la noción geocéntrica del mundo.

Sin embargo, un texto de gran preeminencia para la edad media fue "La sintaxis matemática de la astronomía", también llamado el "Almagesto" (Hè Megalè Syntaxis) escrito por Ptolomeo (85 - 165 d.C.). En dicho manuscrito había un interés por la trayectoria de los planetas los cuales armonizaban con los datos geométricos para explicar el movimiento de los astros, abandonando los modelos terrestres (causas conocidas que operan en la tierra) y así explicar los fenómenos celestes. Por ello Ptolomeo elabora un modelo explicativo a través de epiciclos, de excéntrica y de ecuante. Estos modelos dominaron la astronomía desde el siglo II a.C. hasta el siglo XVII cuando fueron abandonados por Kepler (Torreti 2007: 100) y permitían dar cuenta del movimiento retrógrado de los planetas, considerando a su vez que la tierra era redonda. No obstante no construye una teoría sobre el movimiento de los planetas, sino que éste es un conocimiento empírico descriptivo. Como consecuencias posteriores, se señala que los cálculos de Ptolomeo subestimaron el radio de la tierra, lo que habría hecho que Cristóbal Colón (siglos posteriores) errara en el tiempo para dar la vuelta al mundo.

Posteriormente en Europa occidental se adquiere un vuelco en su autodescripción, la ciencia griega se considera pagana, se rechaza la esfericidad de la tierra, y los centros de los sabios se van desplazando. Después de la cuarta cruzada (1204 d.C.) el saqueo de Constantinopla difunde los textos de Aristóteles y en 1210 los textos de dicho autor son prohibidos dada la dificultad de reconciliar el conocimiento astronómico aristotélico con la teología musulmana y cristiana, tal como sugiere Averroes. Sin embargo, Santo Tomás reinstaurará la doctrina aristotélica llenando dicho vacío para, finalmente, desembocar en la concepción astronómica en una

\footnotetext{
${ }^{5}$ Solo por nombrarse algunos casos podemos encontrar los avances de Hiparco de Samos (siglo II a.C.) con respecto al catálogo de estrellas, el cálculo del mes lunar medio y del año solar medio, junto con la precesión del eje de la tierra. La propuesto del poder incorpóreo (momentum) para explicar el problema del proyectil (concepción eleática de la trayectoria de la flecha) y resolver la imposibilidad del infinito de Juan Filopón (siglo VI). Las críticas de Sosígenes (siglo II d. C.) sobre el tamaño aparente de los astros. Las variaciones de la velocidad de los planetas, lo cual es explicado por epiciclos por Heráclides de Ponto (388 aprox. -312 aprox. a.C.) para el Sol, Mercurio y Venus, y por Apolonio (262 aprox. - 190 aporx. a.C.) para el caso de Marte, Júpiter y Saturno. Aristarco de Samos (310 aprox. - 230 aprox. a.C.) con respecto a la primera teoría heliocéntrica (estrellas y sol están en reposo) no muy tomada en cuenta, o Arquímides de Siracusa $(287$ - 212 a.C.) con respecto al razonamiento del límite (aparente "centro de gravedad" para resolver el problema de la estática).
} 
mezcla entre Aristóteles y Ptolomeo, cuya figura por excelencia (que da cuenta del universo de la época) derivó en la imagen de la Divina Comedia de Dante. Por largos años, como se puede apreciar, la religión con su forma teológica garantizaría una descripción unitaria del mundo con una alta posibilidad de superar las inconsistencias (Luhmann 2007: 733), por ello, la astronomía resuelve sus problemas de identidad ajustando sus conocimientos a las nociones teológicas dominantes.

Desde otra perspectiva, Copérnico (1473 - 1543) observa los postulados geocéntricos de Ptolomeo y escribe su libro "Sobre las revoluciones de las esferas celestes" (De revolutionibus orbium coelestium). A través de sus estudios, critica a los modelos de la época por forzar teóricamente fenómenos que no eran comparables y sugiere una coherencia en la teoría. En el siglo XVI se parte de la idea, en términos generales, que debe haber un esfuerzo por restablecer el saber y la habilidad de lo antiguo (Luhmann 2007: 793), por ello, no pareciera ser extraño que prevaleciera en dicha época aún la visión de Aristóteles (en donde se puede observar los modelos de Eudoxo) y la de Ptolomeo. No obstante, la principal crítica al modelo de Ptolomeo, se debía a la existencia del ecuante, el cual consistía en un punto cerca del centro de la órbita de los planetas, en que el centro del epiciclo proponía que las órbitas tuvieses una velocidad constante, permitiendo el cambio de velocidades con respecto a distintas posiciones de su deferente (círculos pequeño en que gira un planeta). La crítica de Copérnico suponía que las esferas rotaban alrededor del Sol (punto medio que no está en los deferentes, sino en el punto medio aproximado), el cual era el centro del universo. Con ello propone un modelo que permite resolver las retrogradaciones de los planetas, la aparente ausencia de paralaje, la traslación de los planetas, entre otros. Pero dado que imagen de la divina comedia fue de tal relevancia para las autodescripciones de la sociedad, problematizar dichas nociones del mundo derivaron, en algunos casos, en escándalo por parte de la cristiandad más conservadora de la época. Un caso de esto puede ser encontrado en Giordano Bruno (1548-1600) quien fue muerto en la hoguera por postular el heliocentrismo, idea que ya venía circulando en aquellos años (Tredwell \& Barker 2004). Por ello, la doctrina copernicana era vista como peligrosa.

Esta noción heliocéntrica del mundo es considerada como uno de los hitos de la ciencia moderna. Sin embargo, es la figura de Galileo Galilei (1564-1642) la que radicaliza la diferenciación entre ciencia y religión defendiendo, por ejemplo, el heliocentrismo de Copérnico en las cortes de 
Florencia y de Roma, y publicando el "Diálogo" (Dialogo sopra i due massimi sistemi del mondo) entre Salviati (portador del heliocentrismo de Copérnico), Simplicio (quien defiende el modelo de Aristóteles-Ptolomeo) y Sagredo (aparente visión neutral) en donde no le creen su pretendida neutralidad, por ello el 22 de junio Galileo es juzgado por ser "vehementemente sospechoso de herejía". Sin embargo, con este juicio simbólicamente se problematiza la diferenciación del código de la verdad en la sociedad de la época, entre una verdad religiosa y una verdad científica.

Con respecto a la socio-evolución en la diferenciación de la astronomía, se puede señalar que Galileo no solo usa el tradicional método científico de combinar lógica con observación, sino que además sustenta sus argumentos matemáticamente y utiliza el telescopio. Esta combinación será de relevancia para la ciencia moderna, pues a juicio de Luhmann, "Los éxitos tecnológicos de la ciencia explican, hasta dónde puede llegar su prestigio" (1996: 447), lo cual puede observarse en la creciente proliferación y tecnificación de telescopios en la sociedad contemporánea, y en la enorme influencia que tuvo el autor en aquella época. Con ello, Galileo descubre que existían más estrellas de las que se pensaban (como ejemplo en la constelación de Orión), la luna no era lisa sino rugosa (no había una esfericidades perfecta, ordenada y sana como pensaban los aristotélicos), observa nebulosas como grupos de estrellas y observa lunas en júpiter (la luna no era la única excepción de rotación con respecto al sol). Con los telescopios se amplifica la variación del sistema, ampliando la posibilidad de lo nuevo. Como señala Luhmann (2007: 795): “Entonces, ¿de dónde viene, pues, la originalidad, la inspiración, lo nuevo? Probablemente haya que contestar: del unmarked space, del mundo-sin-observar y sin señalarse", o sea, de cuerpos celestes que no habían sido observados.

Sin embargo, la controversia queda finalmente resuelta cuando Newton propone una explicación dinámica del sistema planetario kepleriano ${ }^{6}$ basado en el concepto de gravedad creado por él (Torreti 2010: 80), no obstante, es en la complejidad del "Principia" (Philosophia naturalis principia mathematica) en donde se vuelve a reconciliar las leyes naturales de la Tierra con la de los cuerpos celestes (al igual que como lo hizo Aris-

\footnotetext{
${ }^{6}$ Por asuntos de espacio no hemos considerado la visión kepleriana del mundo en donde dicho autor, influenciado por los pitagóricos, buscó una ley que rigiera las distancias de los planetas y consumó la astronomía de Copérnico (Torreti, 2007: 123). En el primer libro publicado, realiza un diagrama en donde los cinco poliedros regulares encajan con las esferas concéntricas, de tal forma de reproducir los radios relativos de las órbitas planetarias del sistema copernicano. A diferencia de los modelos cinemáticos, Kepler considera que se requiere una explicación dinámica del sistema que explique las fuerzas que causan los movimientos de sus partes. Se señala que este autor fue relegado por Newton, pero sus tres leyes le brindan una base empírica a su teoría.
} 
tóteles). De ello se intenta explicar la caída de los cuerpos que no tienden naturalmente a su centro (como pensaba Aristóteles) sino que esto se produce por una acción local, a través de una fuerza impresa desde fuera. De todos modos, uno de los aspectos cruciales de Newton es en relación con la noción de proyectil en donde concibe a la Luna como un proyectil con una alta velocidad horizontal y que cae verticalmente desde dicha distancia que se mantiene en órbita en vez de bajar al suelo. Incluso radicaliza su postura asumiendo que existe una fuerza de gravedad tendiente a todos los planetas, en donde cada planeta gravita hacia todos los otros y que todos los cuerpos gravitan hacia cada planeta, con esta idea se descarta el centro inmóvil de atracción no existente en la naturaleza.

Dicho periodo (entre 1637-1687) es reconocido como la fuente de las matemáticas modernas (Bell 2012) y es el auge de la relación entre matemática y naturaleza. Tal como la famosa frase que Galileo señala en el Saggiatore: "La naturaleza está escrita en lenguaje matemático". Al respecto, Luhmann (2007: 785) reconoce dicha cualidad en las autodescripciones de la temprana sociedad moderna como sustrato de certidumbre que únicamente se podía emplear al interior de la ciencia, a través de ecuaciones matemáticas que permitían comprender la naturaleza. Por ello, las autodescripciones de la sociedad que estaban emergiendo, se condicen espacio-temporalmente con ciertas estructuras que comienzan a dar origen a la sociedad mundial como singularidad histórica. Para Stichweh (2012b: 5-7) el origen de la sociedad mundial comienza en los siglos XV y XVI como un proceso en expansión consolidado principalmente vía colonización, y que produce la génesis de la sociedad moderna producto del proceso de formación de tres estructuras: la diferenciación funcional, la generación de membresías organizacionales y las tecnologías comunicativas.

Para el caso de la astronomía, coincide en estas tres innovaciones para la formación de estructuras en la sociedad mundial. En primer lugar en la sociogénesis de su proceso de diferenciación funcional: "la ciencia, en todo caso, en el siglo XVII se disuelve la simbiosis entre ciencia y magia que duró más de dos mil años" (Luhmann 1996: 460) sin embargo Stichweh (2012b: 5) señala que la diferenciación de la ciencia se da entre los siglos XVI al XVIII. En segundo lugar se puede señalar la membresía organizacional dado que en dicho siglo emergen las "academias" (como necesidades especiales de grupos específicos) (Luhmann 2007: 755) junto con extenderse globalmente las relaciones sociales a través de conversaciones 
que forman estructuras en red de comunidades epistémicas profesionales y científicas (Stichweh 2012a: 26). Como ejemplo: La Royal Society (fundada en Londres en donde Newton fue presidente), la Académie des Sciences (París, en donde participaron Mersenne, Descartes y Mydorge) o la Societät der Wissenschaften (Berlín cuyo primer presidente fue Leibniz); y finalmente los conocimientos astronómicos se difunden con la ayuda de la imprenta lo cual produce, según Luhmann (2007: 790), un desplazamiento de la noción de naturaleza hacia una semántica que se reflexiona a sí misma producto de la disposición de libros al alcance de todos los interesados como consecuencia de la imprenta. Estos ejemplos permiten considerar la hipótesis de que coinciden las innovaciones de la sociedad mundial con la diferenciación de la ciencia moderna.

\section{COMENTARIOS FINALES}

Si la ciencia es un sistema funcionalmente diferenciado, aquí hemos sugerido algunas claves para rastrear cómo fue el proceso socioevolutivo de la ciencia moderna a través de un proceso de identidad propio de la astronomía. No obstante, dado dicho problema, la ciencia ha cambiado la forma de la autodescripción de la sociedad y ésta se imbrica espacio-temporalmente con los mecanismos que dan origen a la sociedad mundial (tecnologías de la comunicación, membrecía organizacional, diferenciación funcional).

En otras palabras, pareciera ser que tal como se observa en la ciencia diferenciada del siglo XVII, la ciencia entrega un criterio idealizado fundante en la sociedad. Pues si bien Luhmann (2007: 706) considera que la sociedad está destinada a practicar una autorreferencia sin criterio, considera que en las teorías de la reflexión, por ejemplo, los sistemas funcionales suelen describirse en la mayoría de las veces exigiéndoles "cientificidad", mientras que en las sociedades premodernas valían criterios religiosos.

La astronomía podría ser un caso de este privilegio, en donde se ha transitado de una posición geocéntrica del mundo hacia una heliocéntrica como un problema específico de identidad en dicha disciplina, que se generaliza a otras dimensiones de la sociedad a través de una auto-implicación cognitiva en las descripciones de la sociedad.

Finalmente, la ciencia cambia nuestra imagen de mundo, y se situó en una posición privilegiada en la auto-descripción de la sociedad, desde su sistema funcionalmente diferenciado como en nuestra vida 
cotidiana, el cual fue posible tras un largo proceso socio-evolutivo de formación del cual se puede apreciar una distinción semántica concordante con los procesos de identidad propios de la astronomía. Lo que hace plausible la predominancia de la clausura autorreferencial de la disciplina astronómica frente a las autodescripciones del siglo XVII, produciendo un proceso de redundancias y expansión aceleradas de las comunicaciones, en torno a la distinción religión/ciencia con respecto al movimiento de los astros y sus posiciones relativas.

Aquí hemos usado la astronomía para hacernos algunas ideas al respecto, en la transición de una noción geocéntrica del mundo a otra heliocéntrica. Sin embargo, se podría diferenciar si ésta noción general de ciencia aplica para otras disciplinas, cuáles son fenómenos similares al cambio en la autodescripción de la sociedad, indagar en qué medida los sistemas funcionalmente diferenciados exigen dicha "cientificidad", cómo la ciencia es legitimada en la vida cotidiana, entre tantas otros temas por profundizar, y que puedan cambiar la forma de auto-descripción en el horizonte de la sociedad mundial.RM

\section{BIBLIOGRAFÍA}

Africa, T. (1961). Copernicus' Relation to Aristarchus and Pythagoras. Isis, 52(3), 403-409.

Bell, E. T. (2012). Historia de las matemáticas. México D.F.: Fondo de Cultura Económica.

Clarke, L. (1962). Greek Astronomy and Its Debt to the Babylonians. The British Journal for the History of Science, 1(1), 65-77.

Goldstein, B. \& Bowen, A. (1983). A New View of Early Greek Astronomy. History of Science Society, 74(3), 330-340.

Kahn, C. (1970). On Early Greek Astronomy. The Journal of Hellenic Studies, 90, 99-116,

Kuhn, T. (2010). La estructura de las revoluciones científicas. México D.F.: Fondo de Cultura Económica.

Luhmann, N. (2007). La sociedad de la sociedad. México D.F.: Herder.

Luhmann, N. (2009). ¿Cómo es posible el orden social? México D.F.: Herder.

Luhmann, N. (1996). La ciencia de la sociedad. México D.F.: Herder.

Torreti, R. (2010). Estudios filosóficos 2007-2009. Santiago de Chile: Editorial Universidad Diego Portales.

Torreti, R. (2007). De Eudoxo a Newton: Modelos matemáticos en la filosofía natural. Santiago de Chile: Editorial Universidad Diego Portales.

Tredwell, K. \& Barker, P. (2004). Copernicus' First Friends: Physical Copernicanism from 1543 to 1610. Filozofski vestnik, 25(2), 143-166. 
Stichweh, R. (2012a). El concepto de sociedad mundial. Génesis y formación de estructuras de un sistema social global. En H. Cadenas, A. Mascareño \& A. Urquiza (Eds), Niklas Luhmann y el legado universalista de su teoría: Aportes para el análisis de la complejidad social contemporánea (pp. 23-48). Santiago de Chile: RIL Editores.

Stichweh, R. (2012b). En torno a la génesis de la sociedad mundial: Innovaciones y mecanismos. Revista Mad - Universidad de Chile, 26, 1-16.

\section{SOBRE EL AUTOR}

Alejandro Espinosa Rada es Sociólogo por la Universidad Alberto Hurtado, Chile y Candidato a Magíster en Sociología por la Pontificia Universidad Católica de Chile. Becario Conicyt. Entre sus líneas de trabajo se encuentran: sociología de la ciencia, estudios sociales de la ciencia, la tecnología y la innovación, sociología de las organizaciones.

\section{CONTACTO}

Instituto de Sociología

Pontificia Universidad Católica de Chile

Av. Vicuña Mackenna 4860,

Casilla 306, Correo 22, Macul,

Santiago

Chile

anespinosa@uc.cl

Recibido: Marzo 2014

Aceptado: Julio 2014 